\title{
Nasolabial Flap in the Management of Oral Submucous Fibrosis
}

\author{
${ }^{1}$ Vaishali Jamdade, ${ }^{2}$ Sunil Sharma, ${ }^{3}$ Punit Chitlangia, ${ }^{4}$ Amit
}

\begin{abstract}
Oral submucous fibrosis (OSMF) is a premalignant condition associated with restricted mouth opening. Oral submucous fibrosis is poorly understood and unsatisfactorily treated disease. Serious complication is the risk of development of oral carcinoma. Different treatment modalities like medical, surgical, physical therapy or combination are used to improve the condition of the patient. Surgical treatment is usually indicated in late and irreversible stage of the disease. Successful use of nasolabial flap in the management of OSMF is presented.
\end{abstract}

Keywords: Nasolabial flap, Oral submucous fibrosis, Premalignant condition.

How to cite this article: Jamdade V, Sharma S, Chitlangia P, Amit. Nasolabial Flap in the Management of Oral Submucous Fibrosis. J Mahatma Gandhi Univ Med Sci Tech 2016;1(1):27-29.

\section{Source of support: Nil}

Conflict of interest: None

\section{INTRODUCTION}

Schwartz coined the term "atrophica idiopathica mucosa oris" to describe an oral fibrosing disease. He observed same in five Indian women from Kenya. ${ }^{1}$ Later on Joshi termed the condition oral submucous fibrosis. ${ }^{2}$ Oral submucous fibrosis (OSMF) is defined as "an insidious chronic disease affecting any part of the oral cavity and sometimes the pharynx". It is always associated with juxta-epithelial inflammatory reaction followed by fibroelastic changes of the lamina propria with epithelial atrophy leading to stiffness of the oral mucosa and causing trismus and inability to speak". ${ }^{3}$ It is considered as a 'premalignant condition'. The disease is more prevalent in Indian subcontinent. Oral submucous fibrosis is widely prevalent in all age groups and across all socioeconomic strata in India.

The etiology of OSMF is still unclear, although multiple factors have been implicated in its development. Such factors are areca nut chewing, high chili consumption,

\footnotetext{
${ }^{1}$ Postgraduate Student (3rd Year), ${ }^{2}$ Principal and Head, ${ }^{3}$ Reader

${ }^{4}$ Senior Lecturer

${ }^{1-4}$ Department of Oral and Maxillofacial Surgery, Mahatma Gandhi Dental College and Hospital, Jaipur, Rajasthan, India

Corresponding Author: Vaishali Jamdade, Postgraduate Student (3rd Year), Department of Oral and Maxillofacial Surgery, Mahatma Gandhi Dental College and Hospital, Jaipur Rajasthan, India, e-mail: drjanshuman@rediffmail.com
}

genetic, immunological, and nutritional deficiencies, etc. Therefore, there is no definitive treatment. The main aim of the treatment is to relive symptoms, prevent cancer initiation and improve mouth opening. Surgical treatment is usually indicated in late and irreversible stage. It consists of release of fibrous bands followed by use of various grafts. ${ }^{4}$

In 1830, Dieffenbach first used superiorly based nasolabial flaps to reconstruct nasal alae. Nasolabial flap is reliable and economical option for the surgical management of OSMF. ${ }^{5}$

Here, we are presenting a case of OSMF successfully treated by nasolabial flap with 6 months follow-up.

\section{CASE REPORT}

A 40 years old female reported with pain in right buccal mucosa since 15 days. She is also suffering from oral disability due to restricted mouth opening since last 20 years. Interincisal distance was less than $1 \mathrm{~cm}(0.6 \mathrm{~cm})$. Generalized mucosal blanching and fibrotic bands were palpated involving buccal and retromolar mucosa, palate, floor, and tongue. Superficial ulceration with surrounding erythema was present on right buccal mucosa. Provisional diagnosis of late stage OSMF with an ulcer was given. Patient was informed about the premalignant nature of the condition and importance of functionable mouth opening. Inferiorly based nasolabial flap was planned for surgical mouth opening. Incisional biopsy of ulcer after surgical mouth opening was advised to rule out malignant changes.

The operation was performed under general anesthesia with fiberoptic nasal intubation. First, the buccal mucosa was incised transversely from just behind both corners of the mouth extending posteriorly depending upon the spread of the fibrotic bands. The interincisal distance was increased around $4 \mathrm{~cm}$ immediately after release of fibrous bands. Incisional biopsy of ulcer was taken. Nasolabial flaps from the tip of nasolabial fold to corner of mouth were marked bilaterally (Fig. 1). Both flaps were raised in the plane of the superficial musculoaponeurotic system (Figs 2 and 3). The flap was transposed intraorally through a small transbuccal tunnel near the commissure of the mouth, with no tension and the caudal base of the 


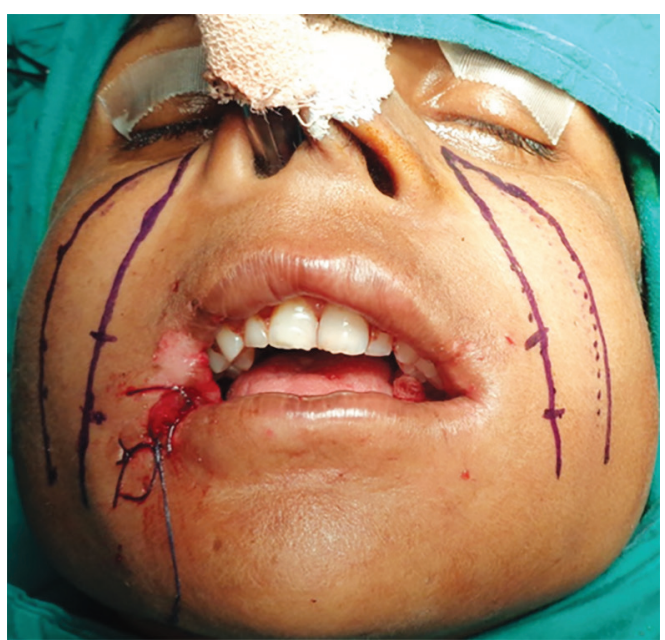

Fig. 1: Surgical markings for nasolabial flap

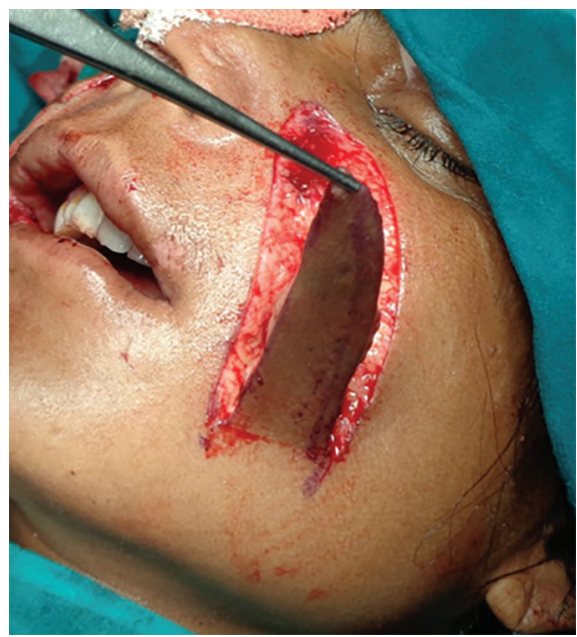

Fig. 3: Nasolabial flap taken from left side

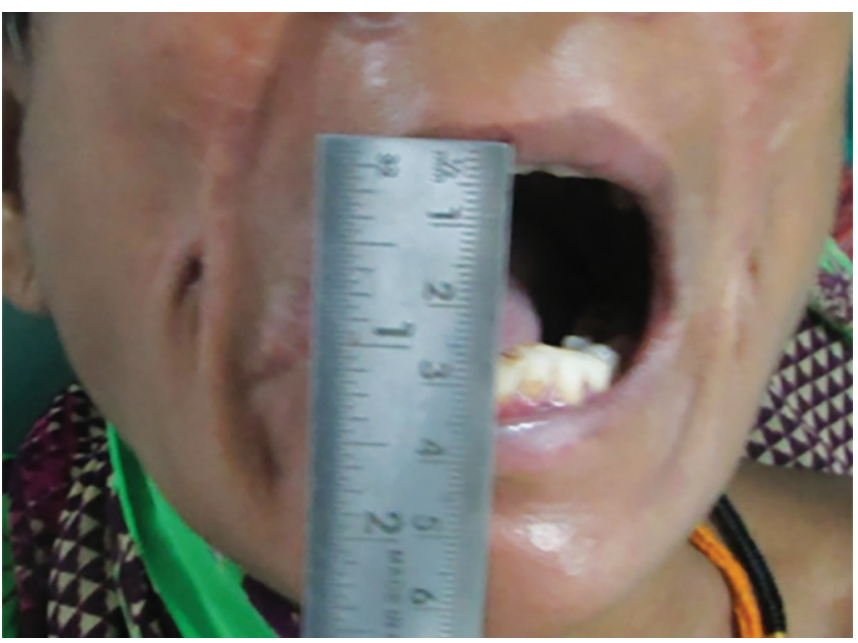

Fig. 5: Six months postoperation

inferiorly based nasolabial flap was deepithelized in a triangular fashion (Fig. 4).

The area of deepithelialization is determined by the area of the defect and so the prepared area for the transbuccal tunnel. Transbuccal tunnel has to be completely covered by the flap. This is the important step of the

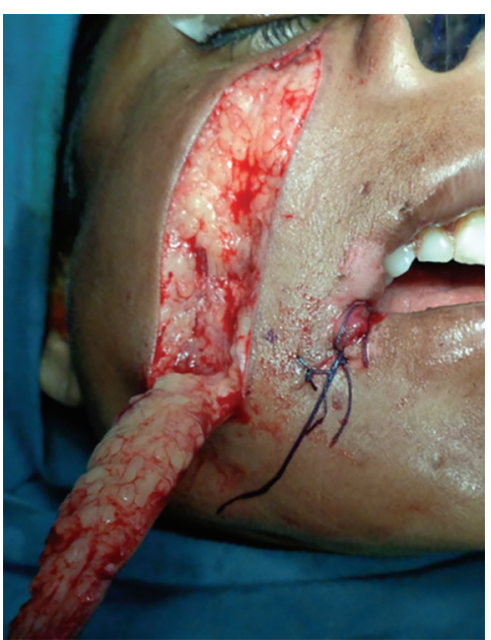

Fig. 2: Nasolabial flap taken from right side

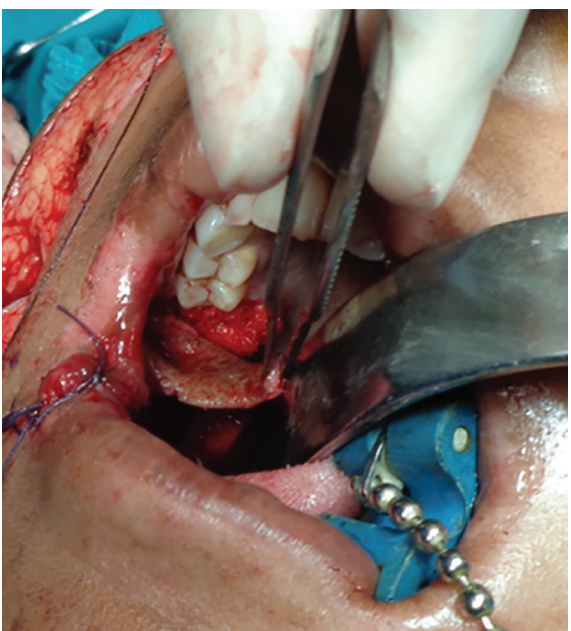

Fig. 4: Flap transposed through a transbuccal tunnel

procedure. The extraoral defect was closed primarily in layers after undermining skin in subcutaneous plane to prevent tension across the suture line. Patient was advised for mouth opening exercises from 4 th postoperative day and to continue same for minimum 6 to 8 months in order to prevent relapse. Six months postoperation interincisal distance was $3 \mathrm{~cm}$ (Fig. 5). There were no further reduction in mouth opening and recurrence of the condition. Histopathological findings of ulcer were negative for malignant changes. Ulcer was healed within 2 weeks after biopsy.

\section{DISCUSSION}

Oral submucous fibrosis is a unsatisfactorily treated disease. Surgical treatment usually aims to relieve trismus, involves incising and releasing the fibrotic areas followed with or without grafting. Various surgical treatments have been proposed by different authors. Each modality has its own advantages and disadvantages. A mucosal graft may be the ideal treatment for oral submucous fibrosis, but is limited in quantity. It can be used for smaller defects. 
Release of fibrous bands and split thickness skin grafting has a high recurrence from contracture. The survival of full thickness skin grafts is questionable. ${ }^{6}$ Esthetics and function achieved with split skin graft were good but showed some degree of relapse due to contracture of the graft. ${ }^{7}$ Borle and Borle reported disappointing results with skin grafting to cover the raw area and used tongue flap to cover the defect. ${ }^{8}$

Tongue flaps cause severe dysphasia, disarticulation, and carry the risk of postoperative aspiration, instability and dehiscence are the common postoperative complication. ${ }^{9}$ In addition, tongue is itself involved in the disease process in some cases. Even though buccal fat pad is found successful in some studies, main drawback is its limited size and cannot be used for larger defects. ${ }^{10}$

However, nasolabial flap can be used to close large defects. It has an edge of close proximity to defect, easily accessibility and modifiable with single sitting surgery resulting in a camouflaged scar. Complications include intraoral hair growth and loss of nasomaxillary crease. A periosteal suture can be used to recreate the crease. ${ }^{11}$ Nasolabial flap has been successfully used in the management of OSMF-induced trismus. ${ }^{5,9,11}$ In our case, patient was satisfied with the treatment with minor complaint of surgical scar.

\section{CONCLUSION}

Inferiorly based nasolabial flap can be used to treat severe trismus and larger defects of OSMF origin.

\section{REFERENCES}

1. Schwartz J. Atrophica idiopathica mucosa oris, in Proceedings of the 11th International Dental Congress, London, UK, 1952.

2. Joshi SG. Submucous fibrosis of the palate and pillars. Ind J Otolaryngol 1953;4:1-4.

3. Pindborg JJ, Sirsat SM. Oral submucous fibrosis. Oral Surg Oral Med Oral Pathol 1966;22:764-779.

4. Canniff JP, Harvey W, Harris M. Oral submucous fibrosis: Its pathogenesis and management. British Dent J 1986;160(12):429-434.

5. Borle RM, Nimonkar PV, Rajan R. Extended nasolabial flaps in the management of oral submucous fibrosis. British J Oral and Maxillofac Surg 2009;47(5):382-385.

6. Khanna, Andrade NN. Oral submucous fibrosis: a new concept in surgical management - report of 100 cases. Int J Oral Maxillofac Surg 1995;24:433-439.

7. Mehrotra D, Pradhan R, Gupta S. Retrospective comparison of surgical treatment modalities in 100 patientswith oral submucous fibrosis. Oral Surg Oral Med Oral Pathol Oral Radiol Endodontol 2009;107(3):e1-e10.

8. Borle RM, BorleSR. Management of oral submucous fibrosis: a conservative approach. J Oral Maxillofac Surg 1991;499(8):788791.

9. Kavarana NM, Bhathena HM. Surgery for severe trismus in submucous fibrosis. Br J Plast Surg 1987;40(4):407-409.

10. Paissat DK. Oral submucous fibrosis. Int J Oral Surg 1981;10(5):307-312.

11. Anisha M, Yogesh S, Preeti K. Use of nasolabial flap in the management of oral submucous fibrosis: a clinical study. People's J Scient Res 2011 Jan;4(1):28-30. 